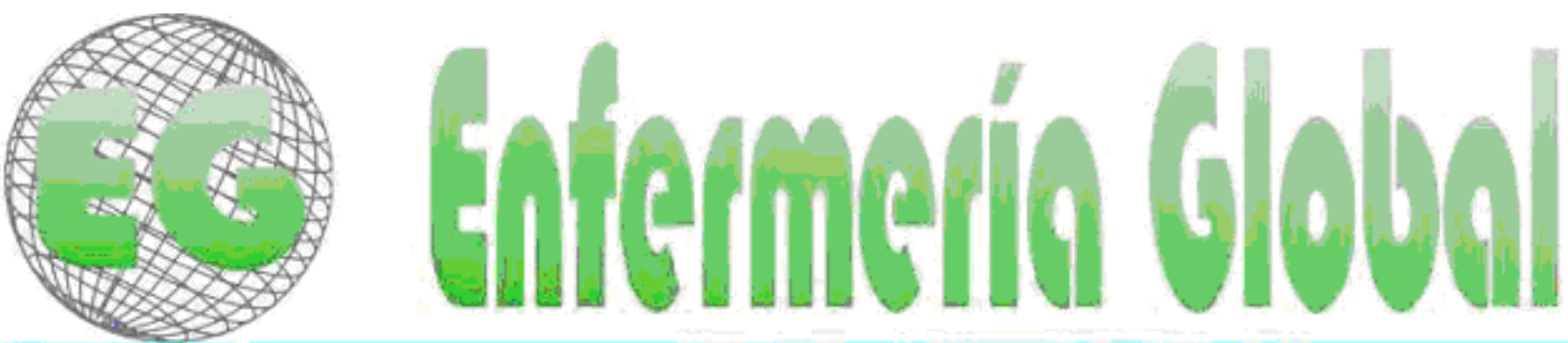

15SN $1696-6+54$

Reuista electrônica cuatrimestral de Enfermeria

$\mathrm{N}^{\circ} 19$

Junio 2010

www.um.es/egloball

DOCENCIA - FORMACIÓN

\title{
CUIDADOS DE ENFERMERÍA A LOS PACIENTES CON FIBROMIALGIA
}

NURSING CARE OF PATIENTS WITH FIBROMYALGIA

\begin{abstract}
${ }^{*}$ Rodríguez Muñiz, RM, **Orta González, MA, *Amashta Nieto, L.
*Enfermera Comunitaria, Consultorio de San Bartolomé de la Torre. Servicio Andaluz de Salud. ${ }^{* *}$ Coordinador de Cuidados de Enfermería Unidad de Gestión Clínica Palos de la Frontera/Mazagón. Servicio Andaluz de Salud. Huelva.
\end{abstract}

Palabras clave: Fibromialgia, Cuidados de enfermería..

Keywords: Fibromyalgia, and Nursing Care.

\section{RESUMEN}

A través de nuestra actividad diaria en el centro de salud hemos detectado una demanda creciente de pacientes que acuden a las consultas de medicina de familia, de enfermería, y de trabajo social, solicitando ayuda ante su situación de salud, mermada por la fibromialgia (FM). Los pacientes con FM se enfrentan al desconocimiento del síndrome por parte de la sociedad y de los profesionales sanitarios.

Ante esta problemática, las enfermeras del Centro de Salud, nos hemos planteado el objetivo de diseñar una actividad educativa para que los profesionales sanitarios puedan actualizar sus conocimientos sobre este síndrome de dolor crónico y para promover entre estos pacientes la adquisición de conocimientos, habilidades y actitudes para poder comprender y aceptar la enfermedad, favorecer la comunicación, enseñar a vivir saludablemente, y fomentar el asociacionismo.

Hemos de actuar sobre ellos de forma individual, con una valoración personalizada, y de manera colectiva, con la realización de talleres de relajación, actividad física, autoestima, higiene postural e intervención psicológica.

Como metodología utilizaremos tanto las exposiciones teóricas como el método interrogativo y las dinámicas grupales.

Esperamos dar a conocer las distintas intervenciones para el manejo de la FM, sobre todo, aquellas que tienen una evidencia fuerte de eficacia.

Y a partir de ahora, para trabajar con estos pacientes, vamos a disponer de un Plan de Cuidados Estandarizados para pacientes con FM. 


\section{ABSTRACT}

Through our daily activities in the health center we have detected a growing demand from patients that go to family medical clinics for treatment, information, nursing and social work, that have been weakened by fibromyalgia (FM). FM patients are confronted with society's and health professional's ignorance of this syndrome.

Faced with this problem, we the nurses at the Health Center, have decided to design an educational activity for healthcare professionals to update their knowledge about this syndrome of chronic pain and to promote among these patients further knowledge, skills and attitudes to understand and accept the disease, improve communication, as well as teaching healthy living and promoting associations.

We must act on them individually with a personalized assessment and collectively, with relaxation workshops, physical activity, self-esteem, healthy posture and psychological intervention.

The methodology we use is both theoretical expositions as well as interrogative method and group dynamics.

We hope to present the different interventions for the management of FM, especially those with strong evidence of effectiveness.

And from now on, to work with these patients, we will have a standardized care plan for patients with FM.

\section{INTRODUCCIÓN}

La Fibromialgia (FM) es una enfermedad de causa desconocida que responde a un incremento de la percepción del dolor a nivel del Sistema Nervioso Central. ${ }^{(1)}$ Es reconocida por todas las organizaciones médicas internacionales y por la OMS desde 1992. Su alta prevalencia la ha convertido en un problema asistencial de primera magnitud. ${ }^{(2)}$

La FM fue definida hace tan sólo 15 años y no porque no hubiera existido antes sino porque no se diagnosticaba como tal. Luego, la definición de este síndrome, basada en criterios reumatológicos, ha provocado que la FM se convierta en un cajón de sastre donde todo cabe: "dolor crónico generalizado de origen desconocido y sin alteraciones orgánicas demostrables según los criterios de los que disponemos actualmente" (Russel, 2001 citado por de Castro Peraza). ${ }^{(3)}$

La FM está considerada como un síndrome doloroso crónico generalizado de más de 3 meses de duración, que afecta al sistema musculoesquelético y presenta una elevada sensibilidad a la presión de determinadas zonas. Este síndrome está acompañado de sueño no reparador y fatigabilidad que afecta de forma severa a la salud. ${ }^{(1)}$

Puede presentarse como única alteración o asociada a otras enfermedades. No tiene causa conocida y antes de llegar al diagnóstico definitivo, la persona con fibromialgia puede haber consultado a diferentes especialistas (digestivo, corazón, traumatología, psiquiatría, reumatología, rehabilitación...).

En 1997, Goldberg y colaboradores investigaron las posibles causas del síndrome sin llegar a obtener resultados concluyentes pero mantuvieron la postura de que se trataba de un proceso multifactorial (neurológico, procesos virales, trastornos de la transmisión y modulación del estímulo doloroso) con consecuencias psico-sociales.

Las investigaciones posteriores han demostrado la existencia de anormalidades bioquímicas, neuroendocrinas, musculares y psicológicas que se asocian con la fibromialgia. Estas anomalías nos permiten entender las alteraciones que presentan los pacientes y establecer un tratamiento adecuado. ${ }^{(4)}$ 
La FM es una dolencia poco conocida y con una importante repercusión sociosanitaria y psicosocial ${ }^{(2)}$. Es una enfermedad frecuente, se estima que la padece entre el 1 y el $4 \%$ de la población, lo que supondría entre 400.000 y 1.600 .000 personas con fibromialgia en España. ${ }^{(5)}$

Los síntomas ${ }^{(5)}$ más frecuentes en estas personas son:

El síntoma más importante de la fibromialgia es el dolor. La persona que busca la ayuda del personal sanitario suele decir "me duele todo". Además del dolor, la fibromialgia puede ocasionar rigidez generalizada sobre todo al levantarse por las mañanas y sensación de hinchazón mal delimitada en manos y pies. También pueden notarse hormigueos poco definidos que afectan de forma difusa sobre todo a las manos.

Otra alteración característica de la fibromialgia es el cansancio que se mantiene durante casi todo el día. Las personas que padecen FM tienen una mala tolerancia al esfuerzo físico. Se encuentran como si se les hubiera agotado la energía, "como si me hubieran dado una paliza". El $70-80 \%{ }^{(5)}$ de las pacientes con fibromialgia se quejan de tener un sueño de mala calidad," me levanto más cansada de lo que me acuesto", empeorando el dolor los días que duermen mal. Los investigadores encuentran que las pacientes con FM se duermen sin problema, pero su sueño al nivel profundo se altera.

Las personas con FM padecen con mayor frecuencia que las sanas ansiedad y depresión, así como jaquecas, dolores en la menstruación, colon irritable, sequedad en la boca y trastornos de la circulación en las manos y los pies.

El Diagnóstico y Tratamiento de la FM son complicados:

La principal dificultad para diagnosticar esta patología es que se desconoce su causa y que no existen alteraciones que se puedan detectar fácilmente para llegar a un diagnóstico rápido y certero.

Cuando un paciente con fibromialgia entra por primera vez a la consulta del médico, él no es el único que va a someterse a examen: también el facultativo se pone a prueba; ya que los síntomas que acompañan a esta enfermedad son indicios compartidos con otras muchas dolencias. Además, para descubrir esta enfermedad, no hay radiografía, escáner ni análisis que valga. De modo que los médicos sólo cuentan con su "ojo clínico" y con el testimonio desesperado del paciente, que a veces es puesto en tela de juicio.

El paciente tiene que presentar dolores generalizados en los 4 cuadrantes del cuerpo más la espalda, por un período de al menos 3 meses, y debe tener dolor en 11 de los 18 puntos sensibles, establecidos para su diagnóstico. ${ }^{(1)}$

Ante la dificultad del diagnóstico y la poca efectividad del tratamiento, muchas personas con FM desconfían del modelo médico hegemónico y recurren cada vez más a las terapias alternativas para dar respuesta a sus problemas de salud. ${ }^{(6)}$ 
En este contexto, la Consejería de Salud de la Junta de Andalucía, elaboró en 2005, con la colaboración de un grupo multidisciplinar de especialistas y los propios pacientes, el Proceso Asistencial Integral, donde se detallan los aspectos básicos de la atención a este tipo de pacientes: quién le atiende, cómo se realiza la asistencia, en qué momento y en qué lugar.

Sin embargo, en la actualidad este proceso está muy poco implantado en Andalucía. Muchos profesionales sanitarios no saben que existe, y los que lo conocen no tienen la formación ni los medios para afrontar las demandas cada vez más crecientes de estos pacientes.

La implantación del citado Proceso podría reducir la variabilidad en la práctica clínica, facilitando el conocimiento de aquellos aspectos asistenciales más idóneos en relación con la evidencia científica actualmente disponible, así como promover la mejora en la utilización racional de los recursos sanitarios y estimular el desarrollo de nuevas líneas de investigación sobre las causas de la enfermedad, su diagnóstico y tratamiento.

En el Proceso Asistencial Fibromialgia se detallan los Planes de Cuidados que debe realizar la enfermería tras el diagnóstico. Pero para ello debemos informarnos y formarnos sobre esta patología. ${ }^{(7-8)}$

La eficacia del tratamiento se encuentra en un abordaje multidisciplinar, uso de fármacos, actividad física, masajes, estiramientos, higiene postural e intervención psicológica. El refuerzo psicológico se basa en la combinación de terapia cognitivo-conductual acompañada de diversas técnicas de relajación.

\section{MATERIAL Y MÉTODOS}

En nuestra actividad diaria en el Centro de Salud hemos detectado una creciente demanda en las consultas de medicina de familia, de enfermería o de trabajo social, de personas con su salud seriamente mermada por esta enfermedad.

Los pacientes con FM se enfrentan al desconocimiento del síndrome por parte de la sociedad y de los profesionales sanitarios.

Ante esta problemática, las enfermeras del Centro de Salud nos hemos planteado el objetivo de diseñar una actividad educativa para que los profesionales sanitarios puedan actualizar sus conocimientos sobre este síndrome de dolor crónico y para promover entre estos pacientes la adquisición de conocimientos, habilidades y actitudes para poder comprender y aceptar la enfermedad, favorecer la comunicación, enseñar a vivir saludablemente, y fomentar el asociacionismo.

Queremos que el profesional sanitario conozca a fondo esta enfermedad para mejorar la calidad de vida de estos pacientes, disminuyendo la carga asistencial que esta enfermedad supone, así como sus costes económicos.

Intentaremos que el paciente participe activamente en su propio proceso de salud" (9). Los profesionales actuaremos como facilitadores, buscando la participación activa de todos los implicados mediante actividades prácticas y dinámicas grupales. 
Para ello, y basándonos en la experiencia de estudios anteriores ${ }^{(10-11)}$ vamos a realizar una serie de Talleres de relajación, actividad física, autoestima, higiene postural e intervención psicológica.

Podemos asignar al paciente a un GAM (Grupo de Ayuda Mutua). Inicialmente pueden reunirse en alguna sala del propio centro de salud, pero posteriormente conviene que lo hagan fuera de él (recursos comunitarios), con la finalidad de "desmedicalizar" la situación y de que pueda servir para que las pacientes "despeguen" en la creación de asociaciones propias. ${ }^{(5)}$

Las enfermeras, además de nuestros conocimientos y" buen hacer", disponemos de una serie de intervenciones para el manejo de la fibromialgia. Entre ellas, las que se ha comprobado que tienen una evidencia fuerte de eficacia son:

El Ejercicio cardiovascular: eficacia que desaparece al cesar la práctica del ejercicio $(12-14)$

La Terapia cognitivo-conductual: mejoría que se mantiene durante meses. ${ }^{(15)}$

Esta terapia se basa en rechazar los pensamientos distorsionados del paciente acerca de sí mismo, de sus experiencias y su futuro y reemplazarlos por pensamientos más exactos y más alegres.

La Educación del paciente: mediante conferencias a grupos, utilizando material escrito, demostraciones. La mejoría se mantiene durante 3-12 meses ${ }^{(5) .}$

La Terapia multidisciplinaria, como ejercicio + terapia conductual cognitiva o educación + ejercicio. ${ }^{(5)}$

Todas estas intervenciones se plasman en nuestro Plan de Cuidados Estandarizados para personas con fibromialgia. ${ }^{(16)}$

\section{Diagnóstico NANDA $0013^{(17)}$ :}

Dolor crónico r/c incapacidad física o psicosocial crónica.

NIC 1400 Manejo del dolor. ${ }^{(18)}$

Actividades:

- Realizar una valoración exhaustiva del dolor que incluya localización, características, aparición/duración, frecuencia, calidad, intensidad o severidad.

- Determinar el impacto de la experiencia del dolor sobre la calidad de vida (sueño, apetito, actividad, función cognitiva, humor, relaciones, trabajo y responsabilidad).

- Proporcionar información acerca del dolor, tiempo que durará y las incomodidades que se esperan debido a los procedimientos.

- Enseñar el uso de técnicas no farmacológicas (relajación, distracción, terapia de juegos, aplicación de calor/frío). 
- Evaluar la eficacia de las medidas de alivio del dolor a través de una valoración continua de la experiencia dolorosa.

NIC 2210 Administración de analgésicos. ${ }^{(18)}$

Actividades:

- Comprobar las órdenes médicas en cuanto al medicamento, dosis y frecuencia del analgésico prescrito.

- Elegir el analgésico o combinación de analgésicos adecuados cuando se prescriba más de uno.

- Enseñar el uso de analgésicos, estrategias para disminuir los efectos secundarios y expectativas de implicación en las decisiones sobre alivio del dolor.

NIC 1320 Acupresión ${ }^{(18)}$

Actividades:

- Determinar contraindicaciones, como contusiones, tejidos con úlceras, infección, estado cardíaco grave y niños pequeños.

- Determinar los resultados deseados.

- Aplicar una presión uniforme sobre el tejido muscular hipertónico buscando el dolor hasta que sienta relajación o se observe la disminución del dolor, normalmente de 10 a 15 segundos.

- Disponer aplicaciones diarias de Acupresión durante la primera semana del tratamiento del dolor.

- Registrar la acción y la respuesta del paciente a la Acupresión.

NIC 6040 Terapia de relajación simple ${ }^{(18)}$

Actividades:

- Explicar el fundamento de la relajación y sus beneficios, límites y tipos de relajación disponibles (terapia musical, meditación y relajación muscular progresiva).

- Considerar la voluntad y capacidad de la persona para participar, preferencias, experiencias pasadas y contraindicaciones antes de seleccionar una estrategia de relajación determinada.

- Crear un ambiente tranquilo, sin interrupciones, con luces suaves y una temperatura agradable, cuando sea posible.

- Enseñar a la persona a adoptar una posición cómoda sin ropas restrictivas y con los ojos cerrados. 
- Inducir conductas que estén condicionadas para producir relajación, como respiración profunda, bostezos, respiración abdominal e imágenes de paz.

- Grabar una cinta sobre la técnica de relajación para uso de la persona, según proceda.

\section{NIC 4400 Terapia musical $^{(18)}$}

- Determinar el interés del paciente por la música.

- Identificar las preferencias musicales del paciente por la música.

- Elegir selecciones de música particularmente representativas de las preferencias del paciente, teniendo en cuenta el cambio de conducta deseado.

- Proporcionar disponibilidad de cintas/discos compactos de música y equipo al paciente.

- $\quad$ NIC 0200 Fomento del ejercicio. ${ }^{(18)}$

Actividades:

- Valorar las ideas del paciente sobre el efecto del ejercicio físico en la salud.-

- Informar al paciente acerca de los beneficios para la salud y los efectos psicológicos del ejercicio.

- Instruir la paciente acerca de la frecuencia, duración e intensidad deseadas de los ejercicios del programa.

- Instruir al paciente en los ejercicios de precalentamiento y relajación adecuados.

- Enseñar al paciente técnicas de respiración adecuadas para maximizar la absorción de oxígeno durante el ejercicio físico.

- Ayudar al paciente a desarrollar un programa de ejercicios adecuado a sus necesidades.

- Realizar un registro de la actividad.

- Reforzar el programa para potenciar la motivación del paciente.

Un objetivo muy asumible es "caminar entre 20 y 30 minutos en terreno llano tres veces por semana al principio y tras un mes, pasar a hacer esta pequeña caminata cada día" (19). Debe intentar no detenerse mientras que camina estos minutos.

Si a la persona no le gusta caminar puede optar por otros ejercicios suaves, pero deberá consultarlo previamente con su enfermera/o.

Un programa adecuado de ejercicio debe incluir un precalentamiento, un programa de ejercicio aeróbico suave que nunca debe empeorar la situación clínica ni la percepción de enfermedad del paciente y una secuencia de relajación con unas series 
de estiramientos que según el grado de afectación deberán ser supervisados o incluso ejecutados por un experto.

NOC: 1605. Control del dolor ${ }^{(20)}$

- 160501. Reconoce factores causales.

- 160503. Utiliza medidas preventivas.

- 160504. Utiliza medidas de alivio no analgésicas.

- 160505. Utiliza los analgésicos de forma apropiada.

- 160511. Refiere dolor controlado.

Diagnóstico NANDA $00069{ }^{(17)}$ :

Afrontamiento inefectivo $\mathrm{r} / \mathrm{c}$ falta de confianza en la capacidad para afrontar la situación.

NIC 5230. Aumentar el afrontamiento ${ }^{(18)}$

Actividades:

- Valorar el impacto de la situación vital del paciente en los papeles y relaciones.

- Valorar la comprensión del paciente del proceso de enfermedad.

- Proporcionar información objetiva respecto al diagnóstico, pronóstico y tratamiento.

- Alentar una actitud de esperanza realista como forma de manejar los sentimientos de impotencia.

- Evaluar la capacidad del paciente para tomar decisiones.

- Tratar de comprender la perspectiva del paciente sobre una situación estresante.

- Animar la implicación familiar, si procede.

- Ayudar al paciente a identificar estrategias positivas para hacerse cargo de sus limitaciones, y a manejar su estilo de vida o su papel necesario en ella.

NIC 5240. Asesoramiento ${ }^{(18)}$

Actividades:

- Establecer una relación terapéutica basada en la confianza y el respeto.

- Establecer metas.

- Proporcionar información objetiva, según sea necesario y si procede.

- Favorecer la expresión de sentimientos. 
- Pedir al paciente/ser querido que identifique lo que puede/no puede hacer sobre lo que le sucede.

- Determinar cómo afecta al paciente el comportamiento de la familia.

- Ayudar al paciente a que identifique puntos fuertes, y reforzarlos.

- Fomentar la sustitución de hábitos indeseados por hábitos deseados.

NIC 5270. Apoyo emocional ${ }^{(18)}$

Actividades:

- Comentar la experiencia emocional con el paciente.

- Ayudar al paciente a reconocer sentimientos tales como la ansiedad, ira o tristeza.

- Escuchar las expresiones de sentimientos o creencias.

- Facilitar la identificación por parte del paciente de esquemas de respuestas habituales a los miedos.

- Permanecer con el paciente y proporcionar sentimientos de seguridad durante los períodos de más ansiedad.

- Proporcionar ayuda en la toma de decisiones.

NIC 5440. Aumentar los sistemas de apoyo. ${ }^{(18)}$

Actividades:

- Determinar el grado de apoyo familiar.

- Implicar a la familia/seres queridos/amigos en los cuidados y la planificación.

NIC 5430. Grupos de apoyo ${ }^{(18)}$

Actividades:

- Determinar el nivel y conveniencia del sistema actual de apoyo del paciente.

- Utilizar un grupo de apoyo durante las etapas transicionales para ayudar al paciente a que se adapte a un nuevo estilo de vida.

- Determinar el objetivo del grupo y la naturaleza del proceso grupal.

- Crear una atmósfera relajada y de aceptación.

- Clarificar desde el principio las metas del grupo y las responsabilidades de sus miembros y de su líder.

- Formar un grupo de tamaño óptimo: de 5 a 12 miembros. 
- Establecer un lugar y una hora para reuniones grupales.

- Vigilar y dirigir la implicación activa de los miembros del grupo.

- Ayudar al grupo a progresar en las etapas de desarrollo grupal: desde la orientación, pasando por la cohesión hasta la conclusión.

NIC 5450. Terapia de grupo. ${ }^{(18)}$

Actividades:

- Elegir los miembros del grupo que estén dispuestos a participar activamente y hacerse responsables de sus propios problemas.

- Determinar si el nivel de motivación es lo suficientemente alto como para beneficiarse de la terapia grupal.

- Ayudar al grupo a trabajar en la resistencia a los cambios.

- Animar a los miembros a compartir cosas que tengan en común unos con otros.

- Confrontar las conductas que amenazan la cohesión del grupo (cansancio, ausencias, socialización extragrupal, formación de subgrupos y chivos expiatorios).

Utilizar el juego de roles y de resolución de problemas, si procede.

NOC: 1603 . Conducta de búsqueda de la salud. ${ }^{(20)}$

- 160301. Hace preguntas cuando es necesario.

- 160306. Describe estrategias para eliminar la conducta insana.

- 160308. Realiza la conducta sanitaria prescrita cuando es necesario.

- 160309. Busca información actual relacionada con la salud.

Diagnóstico NANDA $00070^{(17)}$.

Deterioro de la adaptación r/c la falta de optimismo, no hay intentos de cambiar la conducta y actitud negativa hacia la conducta de salud.

NIC 5430. Grupos de apoyo. ${ }^{(18)}$

NIC 5240. Asesoramiento. ${ }^{(18)}$

NIC 5450. Terapia de grupo. ${ }^{(18)}$

NIC 5230. Aumentar el afrontamiento. ${ }^{(18)}$

NIC 5820. Disminución de la ansiedad. ${ }^{(18)}$ 
Actividades:

- Proporcionar información objetiva respecto al diagnóstico, pronóstico y tratamiento.

- Escuchar con atención.

- Animar la manifestación de sentimientos, percepciones y miedos.

- Instruir al paciente sobre el uso de técnicas de relajación.

NIC 4360. Modificación de la conducta. ${ }^{(18)}$

Actividades:

- Determinar la motivación al cambio del paciente.

- Fomentar la situación de hábitos indeseables por hábitos deseables.

- Animar al paciente a que examine su propia conducta.

- Identificar la conducta que ha de cambiarse (conducta objetivo), en términos específicos, concretos.

- Desarrollar un programa de cambio de conducta.

- Animar al paciente a participar en el registro de conductas.

- Administrar refuerzos positivos en las conductas que han de incrementarse.

NIC 5602. Enseñanza: proceso de enfermedad. ${ }^{(18)}$

Actividades:

- Evaluar el nivel de conocimientos del paciente relacionado con el proceso de enfermedad específico.

- Proporcionar información al paciente acerca de la enfermedad.

- Comentar los cambios en el estilo de vida que pueden ser necesarios para evitar futuras complicaciones y/o controlar el proceso de enfermedad.

- Discutir las opciones de terapia/ tratamiento.

- Instruir al paciente sobre las medidas para prevenir/minimizar los efectos secundarios de la enfermedad, si procede.

- Enseñar al paciente medidas para controlar/minimizar síntomas, si procede.

- Explorar recursos/apoyo posibles, según cada caso.

NOC: 1300. Aceptación: Estado de salud. ${ }^{(20)}$

- $\quad$ 130007. Expresa sentimientos sobre el estado de salud. 
- 130009. Búsqueda de información.

- 130010. Superación de la situación de salud.

- 130011. Toma decisiones relacionadas con la salud.

- 130014. Realización de tareas de cuidados personales

Diagnóstico NANDA00146 ${ }^{(17)}$ :

Ansiedad r/c necesidades no satisfechas y amenaza de cambio: en el rol, en el estado de salud, en el entorno, en los patrones de interacción, en la situación económica.

NIC 5820. Disminución de la ansiedad. ${ }^{(18)}$

NIC 6040 Terapia de relajación simple. ${ }^{(18)}$

NIC 4400 Terapia musical. ${ }^{(18)}$

NIC 5430 Grupo de apoyo. ${ }^{(18)}$

NOC: 1402 . Control de la ansiedad. ${ }^{(20)}$

- 140203. Disminuye los estímulos ambientales cuando está ansioso.

- $\quad$ 140204. Busca información para reducir la ansiedad

- $\quad$ 140207. Utiliza técnicas de relajación para reducir la ansiedad.

- 140209. Refiere incremento de la duración del tiempo entre episodios.

- $\quad$ 140210. Mantiene el desempeño del rol.

- 140211. Conserva las relaciones sociales.

- $\quad$ 140217. Controla la respuesta de ansiedad.

Diagnóstico NANDA00095 ${ }^{(17)}$ :

Deterioro del patrón del sueño r/c. Pensamientos repetitivos antes del sueño, higiene del sueño inadecuada, el patrón de actividad diurna.

NIC 1850. Fomentar el sueño. ${ }^{(18)}$

Actividades:

- Determinar el esquema de sueño/vigilia del paciente.

- Ajustar el ambiente (luz, ruido, temperaturas, colchón y cama) para favorecer el sueño. 
- Animar al paciente a que establezca una rutina a la hora de irse a la cama para facilitar la transición del estado de vigilia al de sueño.

- Ayudar al paciente a evitar a la hora de irse a la cama los alimentos y bebidas que interfieran el sueño.

NIC. Potenciación de la capacidad de hacer frente a situaciones difíciles. ${ }^{(18)}$

Actividades:

- Valorar la comprensión del paciente del proceso de enfermedad.

- Proporcionar información objetiva respecto al diagnóstico, tratamiento y pronóstico.

- Alentar una actitud de esperanza realista como forma de manejar los sentimientos de impotencia.

- Alentar las manifestaciones de sentimientos, percepciones y miedos.

- Ayudar al paciente a identificar estrategias positivas para hacerse cargo de sus limitaciones, y a manejar su estilo de vida.

NOC: 0004 Sueño. ${ }^{(20)}$

- $\quad 00401$. Horas de sueño.

- $\quad$ 00403. Patrón de Sueño.

- $\quad$ 00404. Calidad de sueño.

- $\quad$ 00406. Sueño interrumpido.

- $\quad$ 00407. Hábito de sueño.

- $\quad$ 00408. Sensación de rejuvenecimiento después del sueño.

- 00410. Despertar a horas apropiadas.

Diagnóstico NANDA00093. ${ }^{(17)}$

Fatiga r/c sensación sostenida de agotamiento y disminución de la capacidad para el trabajo mental y físico a nivel habitual.

NIC 0180. Manejo de la energía. ${ }^{(18)}$

Actividades:

- Determinar las limitaciones físicas del paciente.

- Enseñar técnicas de organización de actividades y gestión del tiempo para evitar la fatiga. 
NIC 0200. Fomento del ejercicio. ${ }^{(18)}$

NIC1850. Fomentar el sueño. ${ }^{(18)}$

NIC. Enseñanza: actividad/ejercicio prescrito. ${ }^{(18)}$

Actividades:

- Informar al paciente del propósito y los beneficios de la actividad/ejercicio prescrito.

- Enseñar al paciente cómo controlar la tolerancia a la actividad/ejercicio.

- Enseñar al paciente una buena postura y la mecánica corporal si procede.

NOC 0002 Conservación de la energía. ${ }^{(20)}$

- 000201. Equilibrio entre actividad y descanso.

- 000203. Reconoce limitaciones de energía.

- 000204. Utiliza técnicas de conservación de energía.

- 000205. Adapta el estilo de vida al nivel de energía.

Diagnóstico NANDA $00119^{(17)}$ :

Baja autoestima crónica r/c larga duración de sentimientos negativos hacia uno mismo o sus capacidades.

NIC 5270. Apoyo emocional. ${ }^{(18)}$

NIC 5400. Potenciación de la autoestima. ${ }^{(18)}$

Actividades:

- Animar al paciente a identificar sus virtudes.

- Ayudar al paciente a establecer objetivos realistas para conseguir una autoestima alta.

- Ayudar al paciente a reexaminar las percepciones negativas que tiene de sí mismo.

NOC 1205 Autoestima. ${ }^{(20)}$

- 120501 Verbalizaciones de autoaceptación.

- 120502 Aceptación de las propias limitaciones.

- 120514 Aceptación de críticas constructivas.

- 120519 Sentimientos sobre su propia persona. 


\section{RESULTADOS}

Estudios ya realizados y contrastados sobre cuidados de enfermería a pacientes con FM ${ }^{(21)}$, demuestran una mejora en la calidad de vida de estos pacientes con una disminución significativa del dolor y recuperación progresiva del patrón del sueño.

Ahora disponemos de un Plan Estandarizado de Cuidados de Enfermería para pacientes con FM que nos muestra las intervenciones más eficaces para tratar los distintos problemas detectados en estos pacientes, tras una valoración integral de los mismos.

\section{CONCLUSIONES}

Conocer esta enfermedad, su diagnóstico y su tratamiento, es un deber de todo profesional sanitario implicado en la labor asistencial.

Si el tratamiento farmacológico ayuda a mejorar el dolor, los cuidados de enfermería ayudan a aceptar y manejar esta enfermedad, mejorando la calidad de vida de estos pacientes.

El disponer de un Plan de Cuidados Estandarizados para pacientes con FM y ponerlo en práctica puede ayudar a reducir la variabilidad en la práctica clínica y mejorar la calidad asistencial, brindando a los profesionales la oportunidad de evaluar sus propias acciones.

\section{BIBLIOGRAFÍA}

(1)Collado A, Alijotas J, Benito P et al. Documento de consenso sobre el diagnóstico y tratamiento de la fibromialgia en Cataluña. Med Clin. (Barc) 2002; 118(19): 745-9.

(2)García López A; Campos Sánchez S. Impacto socioeconómico de la fibromialgia. Rev Esp Reumatol 2000; 27:447-449.

(3) Castro Peraza, M Elisa de. La fibromialgia, en el mejor momento de la vida. Index Enferm (Gran). 2007, (56):55-59.

(4) Carmona, L; Gabriel, R; Ballina, J; Laggon, A; Proyecto EPISER; prevalencia de enfermedades reumáticas en la población española. Metodología, resultados del reclutamiento y características de la población. Rev. Esp. Reumatol; 2001, 28(1); 18-25.

(5)Consejería de Salud. Fibromialgia: proceso asistencial integrado, Sevilla, Junta de Andalucía, 2005.

(6) Alvarez-Hernández, E; Casassola-Vargas, J; Lino-Pérez, L; Burgos-Vargas, R; Vázquez-Mellado, J. Frecuencia de uso de medicinas complementarias y alternativas en sujetos que acuden por primera vez al servicio de reumatología. Análisis de 800 casos. Rev. Esp. Reumatología Clínica, 2006, 2 (4); 183-183.

() Busch AJ, Barber KAR, Overend TJ, Peloso PMJ, Schachter CL. Ejercicio para el tratamiento del síndrome de fibromialgia (Revisión Cochrane traducida). En: La Biblioteca Cochrane Plus, 2008 Número 2. Oxford: Update Software Ltd. Disponible en: http://www.update-software.com. (Traducida de The Cochrane Library, 2008 Issue 2. Chichester, UK: John Wiley \& Sons, Ltd).

(7)Miguéliz Beaumont, M Carmen. Fibromialgia, la gran incomprendida. Génesis de un grupo de autoayuda en un centro de salud. Trab Soc y Salud. 2004 mar. (47):547-555. Comunicación. 
(8) Díaz Alonso, JM; Martínez Seras, M; Arribe Guitiérrez, A; de Sanz Velasco, E; Fernández de las Pelas, $\mathrm{C}$ y Fernández Carnero, J. Visión del paciente que sufre del síndrome fibromiálgico. Rev. Esp. Reumatol. 2004 mar-abr. XXI (2):66-71.

(9) Fayos Álvarez, Delia. Participa con la fibromialgia, mejora tu salud. Alicante. 2002. Pp. 43-49. Comunicación.

(10) Bosch Romero, E; Sáenz Moya, N; Valls Esteve, M; Viñolas Valer, S. Estudio de la calidad de vida en pacientes con fibromialgia: impacto de un programa de educación sanitaria. Comment in: Aten Primaria. 2003 Feb 28; 31 (3):204-5; author reply 205. PMID: 12622993. Atencion primaria / Sociedad Española de Medicina de Familia y Comunitaria Jun 2002.

(11) Gil de Gallego, M. D. Programa de cuidados de enfermería en pacientes con fibromialgia. Enferm Científ. 2002 mar-abr. 238-239:57-62.

(12) Busch AJ, Barber KAR, Overend TJ, Peloso PMJ, Schachter CL. Ejercicio para el tratamiento del síndrome de fibromialgia (Revisión Cochrane traducida). En: La Biblioteca Cochrane Plus, 2008 Número 2. Oxford: Update Software Ltd. Disponible en: http://www.update-software.com. (Traducida de The Cochrane Library, 2008 Issue 2. Chichester, UK: John Wiley \& Sons, Ltd).

(13) Richards SCM, Scott DL. Prescribed exercice in people with fibromyalgia: parallel group randomized controlled trial. BMJ 2002; (325):185-188.

(14) Tomas-Carus, P; Gusi, N; Leal, A; García, Y; Ortega-Alonso, A. El tratamiento para la fibromialgia con ejercicio físico en agua caliente reduce el impacto de la enfermedad en la salud física y mental de las mujeres afectadas. Rev. Reumatología Clínica, 2007; $3(1) ; 33-37$.

(15) Manzanera-Escarti, R; Ibáñez-Tarín, C; Sconfalos, S; Arbesi- Prieto, J. Terapéutica en Atención Primaria. Terapia cognitivo-conductual y medicina de familia en el tratamiento. Rev. Semergen, 2007, 33 (8); 425-429.

(16)Unidad de calidad y procesos. Consultoría. Distrito Sanitario Guadalquivir (Córdoba). Plan de cuidados estándar en personas con fibromialgia. 2008. Servicio Andaluz de Salud. Consejería de salud.

(17) NANDA Nursing Diagnoses: Definitions \& Clasification. Versión en español de la obra original en inglés. Madrid. Editorial Elsevier, 2003.

(18) MacCloskey J.C, Bulecheck G.M. Nursing Interventions Clasification (NIC). Versión en español de la tercera edición de la obra original en inglés. Madrid. Editorial Mosby. 2002.

(19) Fernández-Fernández, J; Méndez-Villanueva, A; Sanz-Rivas, D. Fibromialgia y ejercicio físico. Rev. Archivos de Medicina del deporte, 2007, 24 (118), 121-129.

(20) Jhonson, M; Maas, M; Moorhead, S; Nursing Outcomes Classification (NOC). Versión en español de la $2^{\circ}$ edición de la obra original en inglés. Madrid. Editorial Mosby, 2002.

(21) Bosch Romero E, Sáenz Moya N, Valls Esteve M, Viñolas Valer S. Estudio de la calidad de vida en los pacientes con fibromialgia: impacto de un programa de educación sanitaria. Rev. Aten Primaria. 2003 Feb 28; 31 (3): 204-205. 